\title{
Simulation of Weather Impacts on the Wholesale Electricity Market
}

\author{
Bei Zhang ${ }^{1}$, Payman Dehghanian ${ }^{1}$, Mladen Kezunovic ${ }^{2}$
}

\begin{abstract}
This paper investigates the weather-driven performance of electricity markets in the operational time frame. Employing the probability distribution fitting approaches, weather impacts on power generation and electricity demand are modeled and assessed through processing large volume of historical data sets. Several novel weather-impact indices are proposed in this paper to evaluate the vulnerability of the generation as well as the sensitivity of the loads in response to given weather conditions. By incorporating the stochastic weather behavior into power generation and electricity demand modeling, as well as asset reliability constraints, the uncertain aspects of the market operation, such as system costs, locational marginal prices, congestion, reserve price, etc., can be well evaluated by the use of Point Estimation Method (PEM). Numerical experiments on a modified IEEE-RTS system are eventually conducted to validate the effectiveness of the proposed approach and demonstrate the necessity of weather-impact considerations in the electricity market decision making.
\end{abstract}

\section{Keywords}

Asset, electricity market, generation, load, point estimation method, probability distribution fitting method, weather impacts.

\footnotetext{
${ }^{1}$ Department of Electrical and Computer Engineering, $\mathrm{PhD}$ candidates in Power Systems and Power Electronics Group, Texas A\&M University,

College Station, TX 77843-3128, USA

(adele.zhang@tamu.edu, payman.dehghanian@tamu.edu)

${ }^{2}$ Department of Electrical and Computer Engineering, Eugene E. Webb Endowed Professor

Texas A\&M University,

College Station, TX 77843-3128, USA

(kezunov@ece.tamu.edu)
}

\section{Introduction}

To safeguard the provision of a reliable and affordable electricity for decades to come, the electric industry in all the hierarchical levels of generation, transmission, and distribution is required to become more resilient in the face of climate changes [1]. Weather variations, as one of the driving factors in shaping the electricity demand and generation patterns in day to day operations, need to be predicted and incorporated in the power system studies, hoping to reach more resilient solutions for adapting to growing risks and mitigating the possible weather-driven operational concerns and electricity outages [2].

The wholesale electricity market aims at balancing the generation supply and electricity demand in power systems. Quite a few researches are focusing on the impact of weather variations on the performance of the wholesale electricity market. Several general comments on the potential impacts of weather on the PJM energy market, Western Europe's electricity markets and Australian National electricity market are narrated in [3][6]. Reference [6] talks in particular about the impact of extreme weather conditions on the electricity market operations. Possible research directions related to the impact of weather factors on the electricity market are suggested in [7]. Weather-driven planning concerns in the electricity market decision makings are remarked in [8]. Data-driven models are widely adopted in predicting the prices in the electricity market based on various weather inputs [9]-[12]: Heckman correction is adopted in [9]; time-varying parameter (TVP) regression model is utilized in [10]; neural network is applied in [11]; and support vector machines are discussed in [12]. Under such price prediction approaches, however, the weather impact on the wholesale electricity market cannot be fully unfolded since market prices are not solely dependent on the weather information; several other factors such as the economy of the given area also play a critical role. Therefore, by exerting weather impact on the electricity demand and generation, simulations reflecting the

This is an Open Access article distributed in accordance with the Creative Commons Attribution Non Commercial (CC-BY-NC-ND 4.0) license, which permits others to copy or share the article, provided original work is properly cited and that this is not done for commercial purposes. Users may not remix, transform, or build upon the material and may not distribute the modified material (http://creativecommons.org/licenses/by-nc/4.0/) 
electricity market can be conducted. The impact of weather on the electricity market can be better illustrated in this way, since some unrelated factors can be excluded. The impact of weather on the electricity market price, energy exchange, system overall cost with no considerations to the transmission line constraints or assets' performance is the focus in [13]-[18].

Employing the fundamental concept of the Point Estimation Method (PEM), this paper conducts simulation studies to capture the probabilistic impact of weather factors on the wholesale electricity markets, with the driving inputs of weather impact on the power generation and electricity demand assessed through large volumes of historical data. Different from the previous works, the Unit Commitment (UC) market model is adopted with the weather-driven considerations of the transmission line flow constraints and asset reliability. The existent operational uncertainties introduced by a given weather condition, including weather impacts on the transmission congestion, are simulated and analysed as well.

This paper is structured as follows: Section 2 introduces the approach for the weather impact assessment on the electricity generation and demand, and proposes vulnerability and sensitivity indices. Section 3 presents the possible impacts of weather uncertainties on the assets operational reliability. Section 4 introduces the electricity market model and elaborates the weatherdriven simulations of the wholesale electricity markets employing the PEM concept. Numerical experiments and results are presented in Section 5. The contributions of this study are eventually listed in Section 6.

\section{Weather Impacts on Power Generation and Electricity Demand}

The wholesale electricity market is mainly about the balance of two main components: power generation and electric load. Therefore, it is commonsense to first analyze the impacts of weather on these two components based on a large volume of historical data sets, and then investigate the reaction of the electricity market in response to weather variations.

\subsection{Weather Parameters and Data}

The capacity and efficiency of a generating unit, as discussed in [5], have a significant impact on the electricity market performance as the generation capacity indicates the availability of the generation resources and efficiency relates to the cost of power generation. Weather conditions and factors have obviously a huge impact on the renewable sources of power generation such as wind and solar. Moreover, the conventional sources of generation are also directly or indirectly affected by the weather variations. For instance, the availability of the water influences not only the outputs of the hydropower plants, but also the capacity of other types of generation such as coal-fired plants as water would be needed for their cooling towers. Besides, the temperature does have influences on the efficiency of the generation due to the Carnot's theorem [19].

Therefore, the main weather factors focused in this paper range from the water discharge, temperature, humidity, precipitation, and gauge height, for which the corresponding weather data are collected and adopted in this study. Besides, the data on the generation capacity and efficiency is also needed for the model training.

Electricity demand, which is another key player in the electricity market, is also significantly affected by the weather events. There have been numerous studies on the impact of weather on the electric load and load forecast analysis [20]. Accordingly, the weather factors considered in this paper are the cooling degree days (CDD), heating degree days (HDD), temperature, dew point, and wind speed. The weather data as well as the corresponding load demand are collected and adopted in the analysis.

\subsection{Model Training}

The main purpose of the training process is to obtain the conditional distribution $P(y \mid \mathbf{X})$ of a certain parameter, where $y$ is the focused output to be predicted. In the weather-driven analysis of this paper, $y$ can be the generation capacity, generation efficiency, or electricity demand. $\mathbf{X}=\left[x_{1}, x_{2}, \ldots, x_{\mathrm{n}}\right]$ can be the possible factors that may affect the output, where $n$ denotes the number of inputs. In our case, the $\mathbf{X}$ vector contains a set of the weather inputs (the load in the previous 1 and 2 hours should also be included into the input vector $\mathbf{X}$ in the case of the load probability distribution training).

Statistical methods are adopted and samples are taken to estimate the probability distributions. However, not only the outputs with the exact input $\mathbf{X}$, but also the outputs within the range of $\mathbf{X} \pm \Delta \mathbf{x}$ are sampled. The reasons lie in the following: 1) errors do exist when measuring the weather inputs, and therefore, it is not accurate to just sample the outputs with the exact input values of $\mathbf{X}$; 2) sometimes, the exact input combination $\mathbf{X}$ may not exist in the history, and thus, the data around that particular point is used to promisingly approximate its output. This idea is quite similar to the interpolation fundamentals. The sampling process is illustrated in Fig. 1. If the goal is to predict the probability distribution of the output with a set of inputs $\left(P_{1}, Q_{1}, R_{1}\right)$, denoted as the black points in the figure, a cube is considered around the points to sample the historical data for which the inputs are within the range of $\left[P_{1}-\sigma_{1} \times P_{1}, P_{1}+\sigma_{1} \times P_{1}\right],\left[Q_{1}-\sigma_{2} \times Q_{1}\right.$, $\left.Q_{1}+\sigma_{2} \times Q_{1}\right]$ and $\left[R_{1}-\sigma_{3} \times R_{1}, R_{1}+\sigma_{3} \times R_{1}\right]$, where $\sigma_{1}, \sigma_{2}$ and $\sigma_{3}$ are the relatively small factors. In the case of higher number of inputs to be considered, the cube may then turn into a hypercube. The sampled data, denoted in green in Fig. 1, are utilized to assess the probability distribution using certain distribution fitting techniques. In this work, the outputs are assumed to follow the normal probability distributions. By the method of moments, the normal distribution parameters are approximated through the mean $\left(\mu^{\prime}\right)$ and standard deviation $\left(\delta^{\prime}\right)$ of the samples. Hence, the approximation of the probability distribution can be obtained denoted as $P^{\prime}(y \mid \mathbf{X}) \sim N\left(\mu^{\prime}, \delta^{, 2}\right)$.

\subsection{Vulnerability and Sensitivity Indices}

The mean value $\left(\mu^{\prime}\right)$ of a sample can be adopted to predict the output under a certain input $\mathbf{X}$ when a single value, instead of the probability distribution, is needed for prediction. Therefore, the relationship between the output and certain input $x_{i}$ can be calculated by fixing other input 
variables and running the single value prediction while changing the value of $x_{i}$ in a certain range. The slope of the resulting figure denotes the vulnerability or sensitivity of the output to the certain input $x_{i}$ of interest.

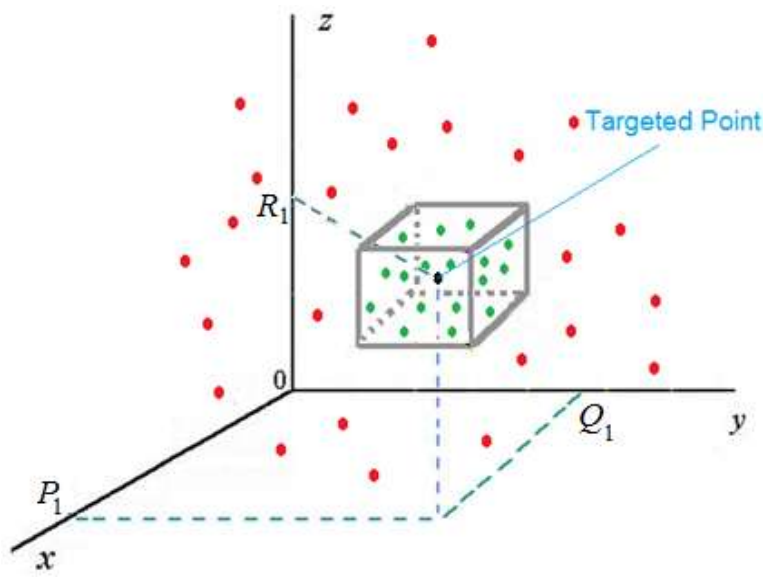

Fig. 1 Illustration of the sampling process.

It would be very helpful if the information is provided on how vulnerable a generating unit feature (capacity or efficiency) is and how sensitive the electricity load is under certain weather conditions $\mathbf{X}$ rather than a certain input $x_{i}$. Suppose that the relationship between the output and input is $y=R(\mathbf{X})$. The Vulnerability / Sensitivity Index under certain input vector $\mathbf{Z}=\left[z_{1}, z_{2}, \ldots, z_{\mathrm{n}}\right]$ can be defined as the total differential of the $R(\mathbf{X})$ at that point, as denoted in (1). Therefore, the following three indices of Generation Capacity Vulnerability Index (GCVI), Generation Efficiency Vulnerability Index (GEVI), and Load Sensitivity Index (LSI) are proposed.

$d y=\left.\frac{\partial y}{\partial x_{1}}\right|_{\mathbf{x}=\mathbf{z}} \cdot \Delta x_{1}+\left.\frac{\partial y}{\partial x_{2}}\right|_{\mathbf{x}=\mathbf{z}} \cdot \Delta x_{2}+\ldots+\left.\frac{\partial y}{\partial x_{n}}\right|_{\mathbf{x}=\mathbf{z}} \cdot \Delta x_{n}$

In the presented case of this study, since the exact relation $R(\mathbf{X})$ is not clearly known, numerical techniques, as shown in (2), to approximate the total differential in (1) are employed.

$$
\begin{aligned}
d y \approx & \left(\mu_{\mathbf{x}=\left[z_{1}+\Delta z_{1}, z_{2} \ldots z_{n}\right]}^{\prime}-\mu_{\mathbf{x}=\left[z_{1}-\Delta z_{1}, z_{2} \ldots z_{n}\right]}^{\prime}\right) \\
& +\left(\mu_{\mathbf{x}=\left[z_{1}, z_{2}+\Delta z_{2} \ldots z_{n}\right]}^{\prime}-\mu_{\mathbf{x}=\left[z_{1}, z_{2}-\Delta z_{2} \ldots z_{n}\right]}^{\prime}\right) \\
& +\ldots+\left(\mu_{\mathbf{x}=\left[z_{1}, z_{2} \ldots z_{n}+\Delta z_{n}\right]}^{\prime}-\mu_{\mathbf{x}=\left[z_{1}, z_{2} \ldots z_{n}-\Delta z_{n}\right]}^{\prime}\right)
\end{aligned}
$$

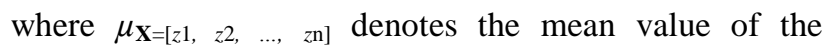
samples around the input vector $\mathbf{Z}=\left[z_{1}, z_{2}, \ldots, z_{\mathrm{n}}\right]$.

\section{Weather Impacts on the Grid Assets}

Assessing the impact of weather variations on the electrical power grid involves a number of considerations. One important aspect is the impact of climate change and weather variations on the performance of the electric equipment. There are huge populations of components (e.g., transformers, circuit breakers, overhead lines, etc.) with the ages of over 25-40 years in service in the USA playing critical roles in power system. Some are located and operated under normal climate conditions and some are exposed to especial climate conditions such as dust, sand, salt deposits, humidity, continuous rain storms, etc. Sudden changes of temperature and other prevailing weather conditions would also affect the components stress and loading, revealing higher risk to the safe and reliable operation of the grid. As a result, predictive indicators on the impact of weather on the performance of such components (with different age and aging mechanisms), as well as other operational parameters would provide a helpful informative knowledge for the operators to foresee a probable mal-operation and decide upon accordingly.

Focusing on the overhead transmission lines in this study, practical engineering judgments and reports reveal that the lines are commonly so well-made that the electrical towers and insulating systems are more likely to fail over time than the overhead lines themselves [21]. Wooden towers begin to rot in 40 years of continuous service, and steel towers would corrode, mostly driven by the weather and environmental drivers. Excessive dirt is also a driving factor for the degradation and deterioration of the insulating structures. Moreover, the severe temperature rise/fall is directly governing the loading of the transmission lines, the increase of power flowing through the lines, and hence leading to extra heat. Such situations may lead to an unpredicted sag condition of the transmission line, which in some cases, could come in contact with the surrounding environment such as trees and other facilities, resulting in a fault causing significant technical and economic consequences.

Fundamentally speaking, the impact of weather is not solely limited to the mal-operation or failure of the components because it may change several weatherdriven parameters and characteristics of the electric components. For instance, temperature variations would result in different resistance values of overhead transmission lines which can significantly affect the maximum power capacity and available transfer capability of the line, accuracy of power flows, state estimation, and other power system applications.

In this study, an online health index for transmission lines is considered, obtained quantitatively through a combination of the condition monitoring data for some line assemblies (e.g., insulators, conductors, etc.) and the inspection data and human judgment for some other compartments (e.g., foundations, tower structures, auxiliaries, etc.) [21]. The health index is a dynamic measure of the line availability for operation and can be updated as new monitoring data arrives. In this study, we also assume different resistance values for a transmission line in different operation hours since the line resistance is a direct function of predicted temperature as demonstrated in (3) [22]:

$$
R_{T_{1}}=R_{T_{0}}\left[1+\alpha\left(T_{1}-T_{0}\right)\right]
$$

where, $\alpha=0.0039[\mathrm{C}]^{-1}$ is the temperature coefficient for the aluminum, and $R_{T 1}$ and $R_{T 0}$ are the line resistances in the temperature $T_{1}$ and $T_{0}$, respectively.

\section{Weather Impact on the Wholesale Electricity Market}

The results of the above-introduced models for the impact assessment of weather factors on the electricity demand, generation, and transmission assets are fed into a 
market clearing simulation to realize how such weather considerations affect the wholesale electricity market.

\subsection{Market Model}

The Unit Commitment (UC) model is utilized here to optimally dispatch the generating units in a given operation time horizon. The model is formulated in (4), where $C_{G i}$ and $C_{R S i}$ are the generation cost and reserve cost of the generating unit $i ; P_{G i}^{t}$ and $P_{R S i}^{t}$ are the output of generator $i$ in energy market and reserve market at time period $t$, respectively; $C_{U i}$ and $C_{D i}$ are the starting up and shutting down costs; $S_{U i}^{t}$ and $S_{D i}^{t}$ are the starting up and shutting down indicators of generator $i$ at time $t ; \mathbf{H}$ is the distribution factor matrix; and $\mathbf{F}$ is the vector of the transmission line limits.

Equation (4.a) is the energy balance equation in which $P_{L}^{t}$ is the electricity demand at time $t$; reserve requirement is cast in (4.b) in which $D_{R S}^{t}$ is the reserve demand at time $\min f=$

$$
\begin{gathered}
\sum_{i \in G} \sum_{t=t_{0}}^{T} C_{G i} p_{G i}^{t}+C_{R S i} p_{R S i}^{t}+C_{U i} s_{U i}^{t}+C_{D i} s_{D i}^{t} \\
\sum_{i \in G} p_{G i}^{t}=P_{L}^{t}, t=1,2, \cdots, T \\
\sum_{i \in G} p_{R S i}^{t} \geq D_{R S}^{t}, t=1,2, \cdots, T \\
P_{G i}^{t}+P_{R S i}^{t} \leq x_{G i}^{t} P_{G i}^{\max }, i \in G, t=1,2, \cdots, T \\
x_{G i}^{t} P_{G i}^{\min } \leq P_{G i}^{t} \leq x_{G i}^{t} P_{G i}^{\max }, i \in G, t=1,2, \cdots, T \\
0 \leq P_{R S i}^{t} \leq P_{G i}^{\max }, i \in G, t=1,2, \cdots, T \\
x_{G i}^{t}-x_{G i}^{t-1} \leq s_{U i}^{t}, i \in G, t=1,2, \cdots, T \\
x_{G i}^{t-1}-x_{G i}^{t} \leq s_{D i}^{t}, i \in G, t=1,2, \cdots, T \\
x_{G i}^{t}, s_{U i}^{t}, s_{D i}^{t} \in\{0,1\}, i \in G, t=1,2, \cdots, T \\
-\mathbf{F} \leq \mathbf{H} \cdot \mathbf{P}_{\mathbf{G}}^{t} \leq \mathbf{F}, t=1,2, \cdots, T
\end{gathered}
$$

$t$. The generation limits of generating units are considered in (4.c)-(4.e), where $P_{G i}^{\min }$ and $P_{G i}^{\max }$ are the minimum and maximum generation limits of generating unit $i$, and $x_{G i}^{t}$ indicates the on/off state of the generating unit $i$ at time $t$. Meanwhile, the on/off states of generating units need to meet the constraints in (4.f)-(4.h). Transmission line constraints are also expressed in (4.i).

When the impacts of weather factors on transmission lines are taken into account, not only the line temperaturesensitive characteristics may change, the lines might not be available all the time due to the weather-driven operational constraints. Therefore, the topology of the system may change accordingly, and an islanding may occur as a result. Once this problem occurs, the UC model will be conducted in each island separately to ensure the generation and load balance is met. Fig. 2 demonstrates the procedure for conducting the market model considering the weather impact and line constraints.

\subsection{Weather-Driven Generation and Load Forecast Inputs to the Market Model}

Section 2 gives the probability distribution of the generation capacity, efficiency and the electricity demand, and that information needs to be fed into the electricity market model, as highlighted in light blue in Fig. 2. The PEM is adopted here to approximate the probability distribution of the market output such as price, system cost, etc. Different from Monte Carlo simulations, which randomly selects the input data and need a huge number of simulation records, the PEM selects some particular input data to simulate the final output. Therefore, the computation burden drastically decreases.

Suppose that the relationship between the electricity market inputs and outputs is expressed as in (5).

$$
\mathbf{F}=\mathbf{g}(\mathbf{Y})
$$

For each input to the electricity market model, suppose a normal probability distribution is assigned and its mean and standard deviation are expressed as $\mu^{\prime}$ and $\delta$ ', respectively. Using the 2-PEM technique, two values will be selected for each input according to equations (6)-

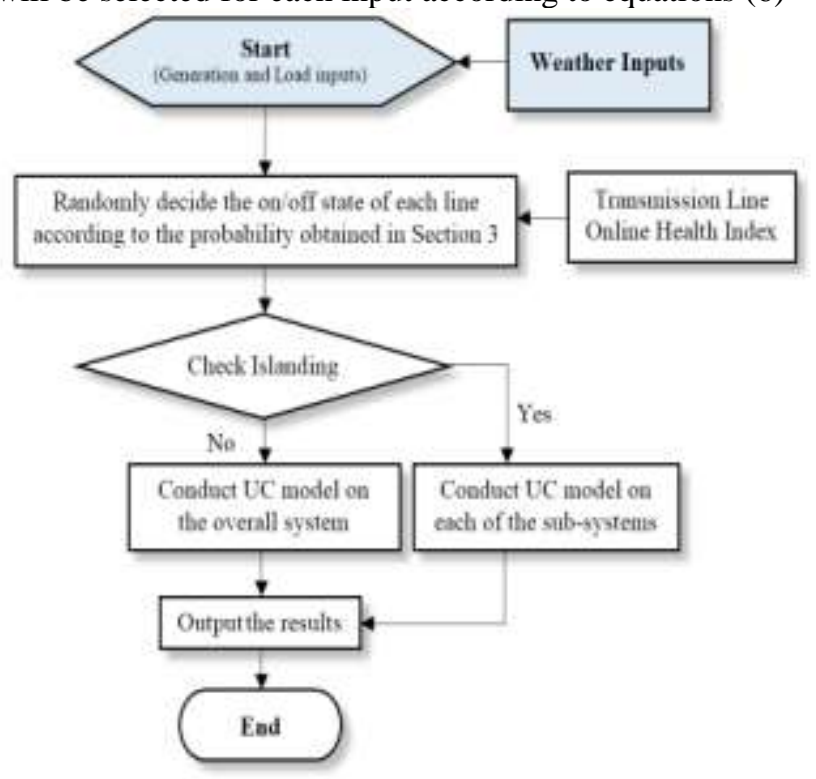

Fig. 2 Flow chart of the weather-driven market model.

(8), where $m$ is the total number of inputs.

$$
\begin{gathered}
y_{i, k}=\mu_{i}^{\prime}+\zeta_{i, k} \cdot \delta_{i}^{\prime}, \quad k=1,2 \\
\zeta_{i, k}=\frac{\lambda_{i, 3}}{2}+(-1)^{3-k} \sqrt{m+\left(\frac{\lambda_{i, 3}}{2}\right)^{2}}, \quad k=1,2 \\
\lambda_{i, 3}=\frac{E\left[\left(y_{i}-\mu_{i}^{\prime}\right)^{3}\right]}{\delta_{i}^{\prime 3}}
\end{gathered}
$$

Having selected the input values, the mean value of the $j^{\text {th }}$ output parameter can be calculated through equations (9)-(11).

$$
\begin{gathered}
E\left(F_{j}\right) \approx \sum_{i=1}^{m} \sum_{k=1}^{2} w_{i, k} \cdot g_{j}\left(\mu_{1}^{\prime}, \mu_{2}^{\prime}, \ldots, \mu_{i}^{\prime}, \ldots, \mu_{m}^{\prime}\right) \\
w_{i, k}=\frac{1}{m}(-1)^{k} \frac{\zeta_{i, 3-k}}{\theta_{i}} \\
\theta_{i}=2 \sqrt{m+\left(\frac{\lambda_{i, 3}}{2}\right)^{2}}
\end{gathered}
$$

Accordingly, the standard deviation of the $j^{\text {th }}$ output parameter can be obtained through equations (12) and (13).

$$
E\left(F_{j}^{2}\right) \approx \sum_{i=1}^{m} \sum_{k=1}^{2} w_{i, k} \cdot\left[g_{j}\left(\mu_{1}^{\prime}, \mu_{2}^{\prime}, \ldots, \mu_{i}^{\prime}, \ldots, \mu_{m}^{\prime}\right)\right]^{2}
$$




$$
\delta_{F j}=\sqrt{E\left(F_{j}^{2}\right)-\left[E\left(F_{j}\right)\right]^{2}}
$$

\section{Numerical Experiments}

\subsection{Test System and Data Sources}

The electricity demand data comes from the PJM data sources [23], and the generation data are borrowed from the U.S.A Environmental Protection Agency (EPA) website [24]. The weather and water data are obtained from the Iowa Environmental Mesonet (IEM) [25] and the U.S. Geological Survey (USGS) web pages [26], respectively. The aforementioned types of data corresponding to the yearly time horizon 2014 are adopted to conduct the model training of the weather impact on the power generation and electricity demand.

The analysis of the weather impact on the wholesale electricity market is applied on a modified 24-bus test system, IEEE RTS, with the installed capacity of 3405 MW through 33 generating units serving the load through 38 transmission lines. All the generating units of the system are assumed to be coal-fired generators, and the loads are assumed to follow the pattern corresponding to the zone EKPC of the PJM system. Other details on the system configuration and parameters can be found in [27]. Two scenarios are considered and compared: 1) weatherdriven market operation with no transmission line constraints (i.e., constraint (4.i) is ignored); and 2) transmission line reliability and line flow constrains are considered in the weather-driven market operation.

\subsection{Training of the Weather-Driven Generation and Load Forecasts}

Fig. 3 illustrates the result of probability density function of the generation efficiency, calculated according to Section 2.2 based on the available historical data, when temperature is ranging from $17{ }^{\circ} \mathrm{F}$ to $100{ }^{\circ} \mathrm{F}$, while humidity is $90 \%$, precipitation is 0 (inches), gauge height is $3.42(\mathrm{~m})$ and water discharge is $6990\left(\mathrm{f}^{3} / \mathrm{sec}\right.$.).

Fig. 4 illustrates the relationship between the electricity demand and the temperature change in 4 cases. For the reference day morning, the CDD is 30, HDD is 0 , dew point is $19\left({ }^{\circ} \mathrm{F}\right)$, wind speed is $0(\mathrm{~m} / \mathrm{s})$, and the load in the previous two hours are $1842 \mathrm{MW}$ and $1856 \mathrm{MW}$; for the windy day, wind speed is $10(\mathrm{~m} / \mathrm{s})$; for the dry day, dew point is $5\left({ }^{\circ} \mathrm{F}\right)$; for the night, the load in the previous two hours are $1658 \mathrm{MW}$ and $1752 \mathrm{MW}$. In contrast with the peak load, the load decreases when the temperature increases. This observation is justified as this situation usually appears in the morning/night of the spring or autumn when the day temperature fluctuates a lot.

The relationship between the capacity of a coal-fired power plant and the water discharge is demonstrated through 3 cases in Fig. 5. For the reference day, the temperature is $73{ }^{\circ} \mathrm{F}$, humidity is $85 \%$, precipitation is 0 (inches), and gauge height is $3(\mathrm{~m})$; for the dry day, humidity is $20 \%$; for the hot day, temperature is $100{ }^{\circ} \mathrm{F}$. The maximum capacity of the generator is $300 \mathrm{MW}$. The vulnerability of generation capacity to the weather variations is tabulated in Table 1. It can be observed that the generation capacity becomes less vulnerable to the weather changes as the temperature decreases, probably due to the possible increase of generation efficiency. Also, the vulnerability greatly decreases when the water becomes more abundant. The load sensitivity results to weather variations are presented in Table 2.

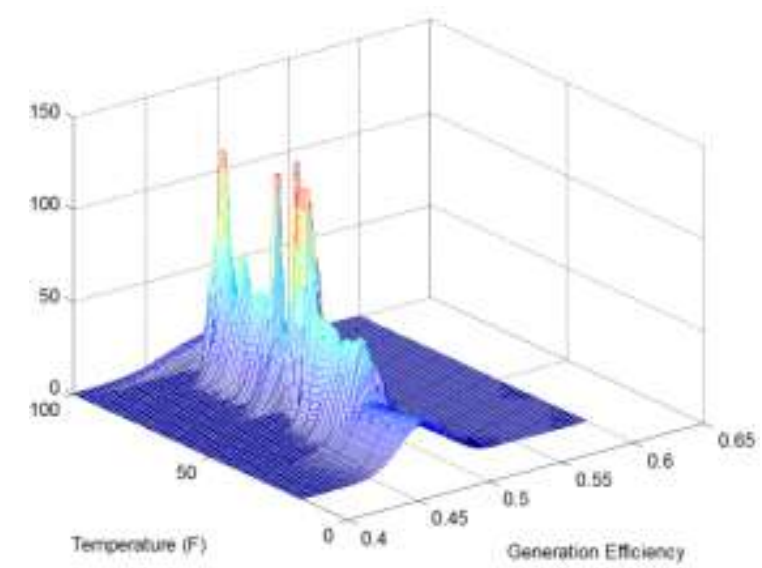

Fig. 3 Probability density function of generation efficiency with the temperature input ranging from $17^{\circ} \mathrm{F}$ to $100^{\circ} \mathrm{F}$.

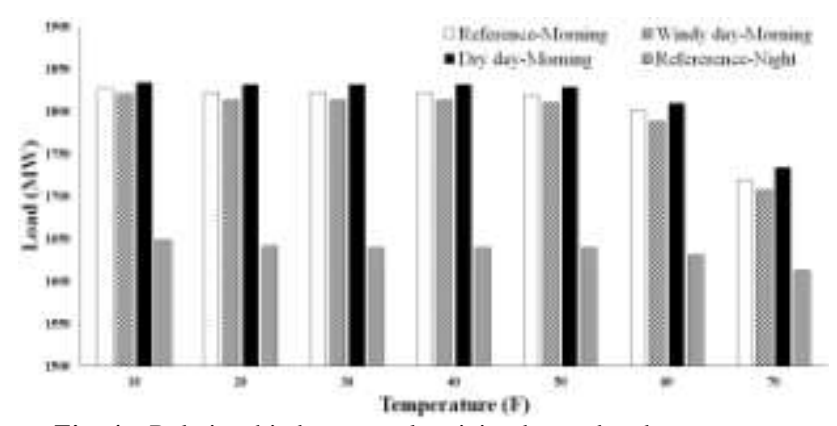

Fig. 4 Relationship between electricity demand and temperature.

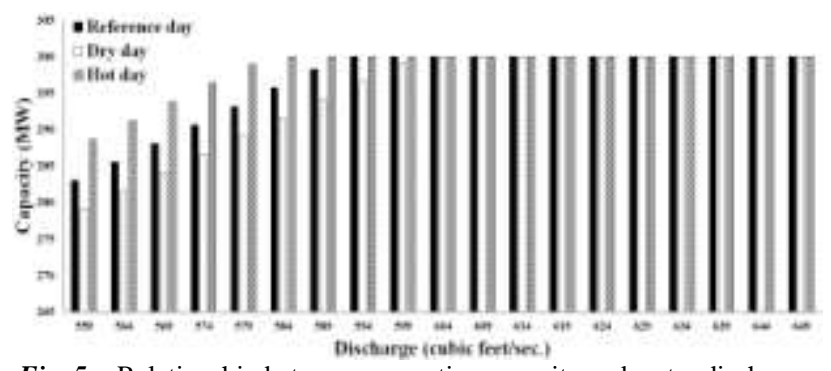

Fig. 5 Relationship between generation capacity and water discharge.

Table 1 Generation capacity vulnerability index

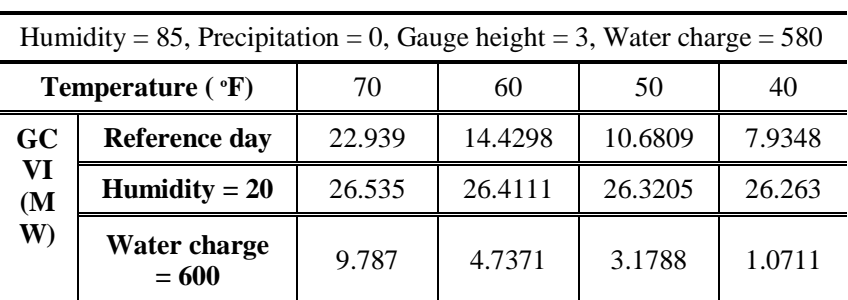

Table 2 Load sensitivity index

$\mathrm{CDD}=30, \mathrm{HDD}=0$, Dew point $=19$, Wind speed $=0$,

Load $(\mathrm{t}-1 \mathrm{~h})=1842 \mathrm{MW}$, Load $(\mathrm{t}-2$ hour $)=1856 \mathrm{MW}$

\begin{tabular}{c|c|c|c|c|c|c}
\hline \multicolumn{2}{c|}{ Temperature $\left({ }^{\circ} \mathbf{F}\right)$} & 15 & 25 & 35 & 45 & 55 \\
\hline \hline \multirow{2}{*}{$\begin{array}{c}\text { LSI } \\
(\mathbf{M}\end{array}$} & $\begin{array}{c}\text { Reference } \\
\text { day }\end{array}$ & 46.259 & 30.051 & 68.142 & 48.394 & -28.32 \\
\cline { 2 - 7 } W) & Dew point & 29.610 & 57.084 & 57.511 & 29.011 & - \\
\hline
\end{tabular}




\begin{tabular}{c|c|c|c|c|c|c}
\hline $\mathbf{= 5}$ & & & & & 43.027 \\
\cline { 2 - 6 } & $\begin{array}{c}\text { wind } \\
\text { speed = 10 }\end{array}$ & 21.957 & 62.723 & 51.298 & 79.235 & - \\
30.327 \\
\hline \hline
\end{tabular}

\subsection{Weather Impact on the Wholesale Electricity Market}

The 24-hour UC is conducted based on the hourly weather information in a 24-hour time horizon, with the training results of the weather impact on the electricity demand, generation, and transmission assets. The PEM method is adopted to estimate the mean and standard deviation of the market outputs. The results of the electricity price in Scenario 1 are illustrated in Fig. 6, where the little circles denote the mean values and the black lines denote the information of standard deviations. Since the transmission line constraints are not considered in Scenario 1, the energy price is unified in the whole system. The results show that weather impact on the electricity market in this scenario is time-variant: the uncertainty of energy price tends to be larger in the first half-day hours, while the uncertainty of the reserve price is larger in the second half-day hours. This is because the load, as demonstrated in Fig. 7, is higher in the first 12 hours of the day and reaches its peak around hours 8 am and $9 \mathrm{am}$. Therefore, some small generating units tend to be switched out from operation after hour 9, and that is why the prices are highly sensitive to the weather during those hours. During the first half day hours, the marginal generating units to provide energy are relatively expensive small generating units, while in the second half day hours, some small generating units become the marginal units to provide reserve. The weather impact on the system cost is illustrated in Fig. 8.

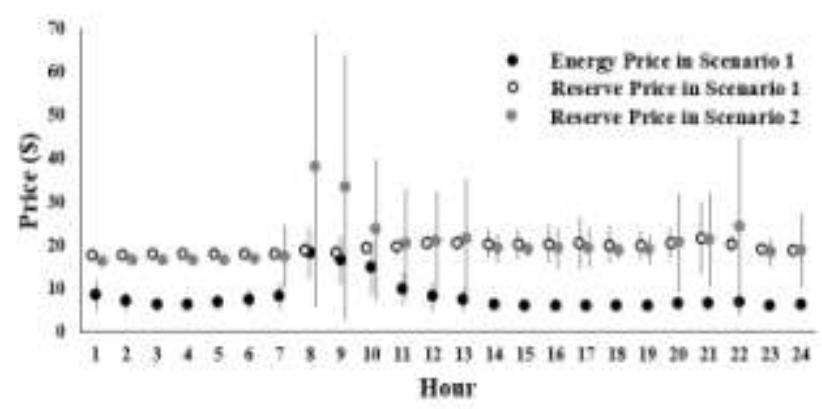

Fig. 6 Illustration of the weather impacts on the market price.

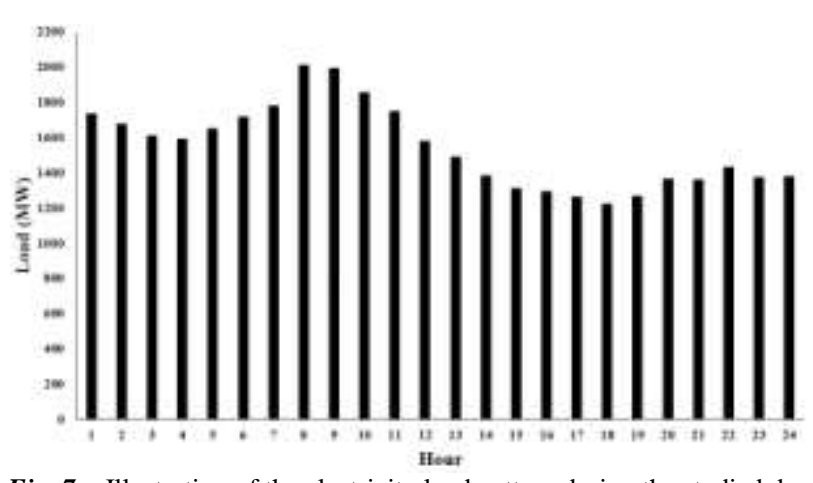

Fig. 7 Illustration of the electricity load pattern during the studied day

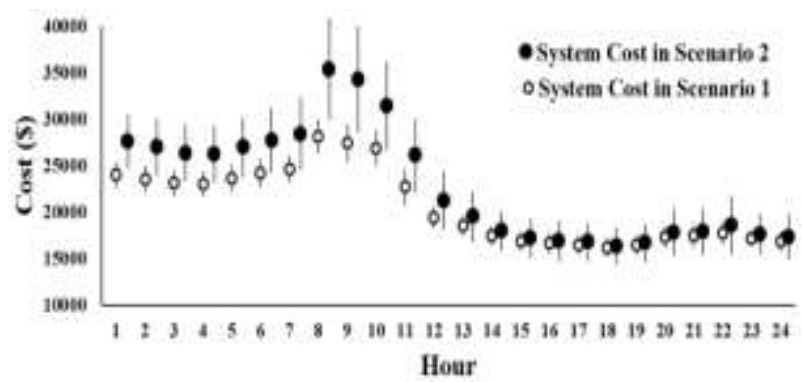

Fig. 8 Illustration of the weather impacts on the system cost.

Weather impact on transmission lines and line flow limits are taken into consideration in Scenario 2. The transmission line health indices of the studied system are depicted in Fig. 9. The uncertainties on the reserve price and system costs in Scenario 2 are illustrated in Fig. 6 and Fig. 8, respectively. In comparison with Scenario 1, the observations reveal a higher amount of uncertainties in Scenario 2. With the inclusion of the transmission line limits, the electricity prices become different at each node of the system. Fig. 10 illustrates the impact of weather variations on the energy price on bus 9 and bus 18, as an example, which have the highest and lowest average energy prices respectively during the 24 hours of interest. Fig. 11 illustrates the weather impact on the transmission line congestions for the transmission lines 7, 12, 13, and 19. The probability of congestion on other lines of the system is very close to 0 and hence is neglected for demonstration. From Fig. 10 and Fig. 11, one can see that the impact of weather variations is not only time-variant, but also space-variant. Some buses and transmission lines are particularly sensitive to the uncertainties imposed by weather.

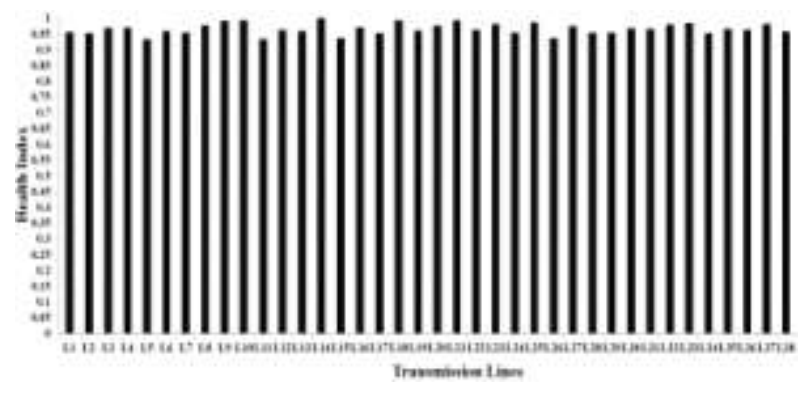

Fig. 9 Transmission line health and reliability indices-IEEE RTS

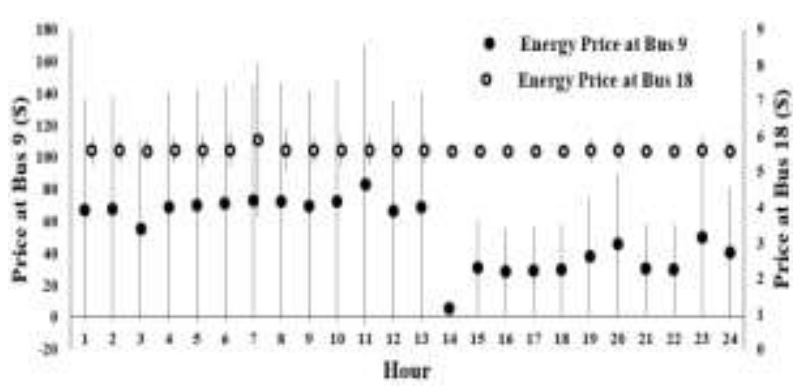

Fig. 10 Illustration of the weather impact on the market price-S2 


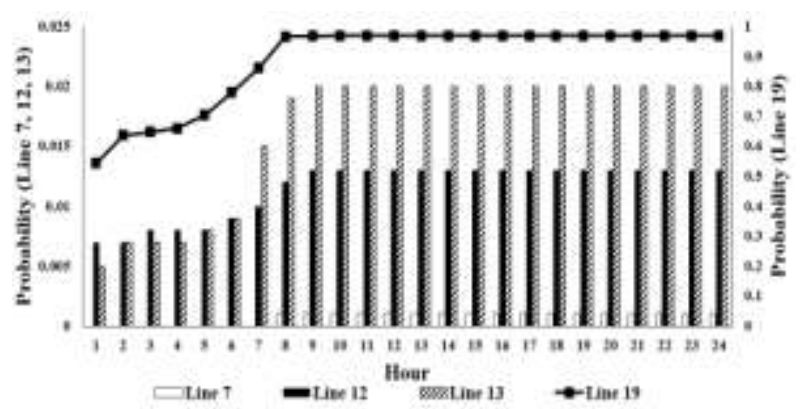

Fig. 11 Illustration of the weather impact on the system transmission line congestions

\section{Conclusion}

The contributions of this work are listed as follows:

- Adequate training of the weather impact on electricity generation and demand based on a large volume of historical data sets is conducted.

- Several innovative indices to evaluate the vulnerability and sensitivity of generation and load to the weather variations are proposed.

- Weather impacts on the performance of the wholesale electricity market are predicted through the PEM capturing the uncertainties introduced by weather.

- Weather-driven market simulations are compared with and without considering the transmission line constraints and reliability to demonstrate benefits of the proposed approach.

\section{References}

[1] M. Davis and S. Clemmer, "Power failure: how climate change puts our electricity at risk and what we can do," Union of Concerned Scientists, 2014.

[2] M. Kezunovic, I. Dobson, Y. Dong, "Impact of Extreme Weather on Power System Blackouts and Forced Outages: New Challenges," Balkan Power Conference, Sibenik, Croatia, September, 2008

[3] PJM Interconnection (2009, Jan.), "Potential Effects of Proposed Climate Change Policies on PJM's Energy Market," URL: http://www.pjm.com/ /medi a/documents/reports/20090127carbon-emissions-w hitepaper.ashx.

[4] PJM Interconnection (2014, May.), “Analysis of Operational Events and Market Impacts During the January 2014 Cold Weather Events," URL: https:// www.pjm.com/ /media/documents/reports/20140509-analysis-ofoperational-events-and-market-impact s-during-the-jan-2014cold-weather-events.ashx.

[5] R. Golombek, S. A. Kittelsen, and I. Haddeland. "Climate change: impacts on electricity markets in Western Europe," Climatic Change, vol.113, pp.357-370, Jul. 2012.

[6] S. Orme and J. Swansson, (2014, Aug.) "Implications of extreme weather for the Australian National Electricity Market: historical analysis and 2019 extreme heatwave scenario," Sapere Research Group, Australia, URL: http://www.industry.gov.au/Energy/ EnergySecurity/nesa/Documents/ExtremeweatherandNEMscenari oreport2014.pdf.

[7] T. Overbye, J. Cardell, I. Dobson, et.al. "The Electric Power Industry and Climate Change: Power Systems Research Possibilities," Power Systems Engineering Research Center, PSERC Publication 07-16, Jun. 2007.
[8] S. C. Nierop, "Envisioning resilient electrical infrastructure: A policy framework for incorporating future climate change into electricity sector planning," Environmental Science \& Policy, vol.40 pp.78-84, Apr. 2014.

[9] R. Handika, C. Truong, S. Trueck, R. Weron, (Aug. 2014) "Modelling price spikes in electricity markets-the impact of load, weather and capacity," Hugo Steinhaus Center, Wroclaw University of Technology, URL: http:// prac.im.pwr.wroc.pl / hugo/RePEc/wuu/wpaper/HSC_14_08.pdf

[10] N. V. Karakatsani, D. W. Bunn, "Forecasting electricity prices: The impact of fundamentals and time-varying coefficients,' International Journal of Forecasting, vol.24, pp.764-785, Oct. 2008.

[11] J. P. S. Catalão, S. J. P. S. Mariano, V. M. F. Mendes, L. A. F. M. Ferreira, "Short-term electricity prices forecasting in a competitive market: a neural network approach," Electric Power Systems Research, vol.77, pp.1297-1304, Aug. 2007.

[12] J. Li, J. Li, "Next-day electricity price forecasting based on support vector machines and data mining technology," in Proceeding of the IEEE Control Conference, pp. 630-633, 2008.

[13] L. Noah, "The Impact of Weather Forecasts on Day-Ahead Power Prices," Senior These, Claremont McKenna College, Claremont, California, 2011.

[14] A. Pechan and E. Klaus, "The impact of heat waves on electricity spot markets," Energy Economics, vol.43, pp. 63-71, May 2014.

[15] C. P. Tung, T. C. Tseng, A. L. Huang, T. M. Liu, M. C. Hu, "Impact of climate change on Taiwanese power market determined using linear complementarity model," Applied Energy, vol.102, pp.432-439, Feb. 2013.

[16] A. Godbole, "Climate change impacts on hydropower and the electricity market: A case study for Switzerland," Master Thesis, Dept. Economics and Oeschger Center for Climate Change Research, University of Bern, Diss. University of Bern, Switzerland, 2014

[17] D. Rübbelke and S. Vögele, "Impacts of climate change on European critical infrastructures: the case of the power sector," Environmental Science \& Policy, vol.14, pp.53-63, Jan. 2011.

[18] M. TH. V. Vliet, S. Vögele, and D. Rübbelke, "Water constraint on European power supply under climate change: impacts on electricity prices," Environmental Research Letters, vol.8, Letter 035010, Jul. 2013.

[19] A. Durmayaz and O. S. Sogut, "Influence of cooling water temperature on the efficiency of a pressurized-water reactor nuclear-power plant," International Journal of Energy Research, vol.30, pp.799-810, Apr. 2006.

[20] D. Fay and J. V. Ringwood, "On the Influence of Weather Forecast Errors in Short-Term Load Forecasting Models," IEEE Transactions on Power Systems, vol.25, no.3, pp.1751,1758, Aug. 2010.

[21] D. Zhang, W. Li, and X. Xiong, "Overhead line preventive maintenance strategy based on condition monitoring and system reliability assessment," IEEE Transactions on Power Systems, vol.29, no.4, pp.1839-1846, April 2014

[22] M. Bockarjova and G. Anderson, "Transmission Line Conductor Temperature Impact on State Estimation Accuracy," in Proceeding of the 2007 IEEE Power Tech. Conference, pp.701706, 2007, Lausanne, Switzerland.

[23] PJM. (n.d.). Estimated hourly load. URL: http://www.pjm.com/markets-and-operations/energy /realtime/loadhr yr.aspx.

[24] U.S.A Environmental Protection Agency (EPA). (n.d.). URL: ftp://ftp.ep a.gov/dmdnlo ad/emissions/hourly/.

[25] Iowa Environmental Mesonet (IEM). (n.d.) ASOS-AWOSMETAR Data Download. URL: http:// mesonet.agron.iastate.edu/request/download.phtml?

[26] U.S. Geological Survey (USGS). (n.d.) Water Watch. URL: http://waterwatch.usgs.gov/?id=ww_ current.

[27] C. Grigg, P. Wong, P. Albrecht et.al., "The IEEE Reliability Test System-1996. A report prepared by the Reliability Test System Task Force of the Application of Probability Methods Subcommittee," IEEE Transactions on Power Systems, vol.14, no.3, pp.1010-1020, 1999 